\title{
Hornhaut-Transplantation in Hochrisikosituationen
}

\author{
Thomas A. Fuchsluger Friedrich E. Kruse \\ Universitätsklinikum Erlangen, Friedrich-Alexander-Universität Erlangen-Nürnberg, Augenklinik, Erlangen, Deutschland
}

\begin{abstract}
Zusammenfassung
In diesem Kapitel werden Vorgehensweisen zur Durchführung von Keratoplastiken in Hochrisikosituationen sowie alternative (z.B. lamellierende) Ansätze vorgestellt. Die historischen und molekularen Hintergründe (Histokompatibilitätsantigene) sowie damit verbundene Risikofaktoren (Neovaskularisation der Hornhaut, Pathologie der Augenoberfläche, Hornhaut-Retransplantationen, Größe des Hornhaut-Transplantats, intraokuläre Chirurgie, Entzündung des vorderen Segments, Herpes simplex, Alter des Patienten) werden erläutert. Das postoperative Management, das die Behandlung
\end{abstract}

von Herpes simplex, die systemische Behandlung von Hochrisikopatienten sowie die Auswahl des Spendergewebes durch HumanLeukozyten-Antigen-Matching einschließt, wird beschrieben. Lamellierende Keratoplastikverfahren werden als Alternativen zur konventionellen perforierenden Keratoplastik besprochen. Die Vor- und Nachteile sowohl der vorderen als auch der hinteren lamellierenden Keratoplastik werden skizziert.

c 2016 S. Karger GmbH, Freiburg

\section{Einleitung}

Hornhaut-Transplantationen sind die am häufigsten und mit dem größten Erfolg durchgeführten Gewebetransplantationen der Welt. Die Gesamtzahl der Transplantationen beträgt Schätzungen zufolge weltweit rund 200000 pro Jahr. In Europa werden jährlich rund 20-25 000 Keratoplastiken durchgeführt [1], in den USA sind es rund 55-60 000 [2].

Das Operationsergebnis hängt jedoch stark vom Zustand der Hornhaut des Patienten vor der Transplantation ab; hier lassen sich risikoarme von risikoreichen Gegebenheiten unterscheiden. Eine risikoarme Situation ist gekennzeichnet durch eine nichtvaskularisierte Cornea und keine Vorgeschichte von HornhautEntzündung in der Gegenwart oder Vergangenheit. In Hochrisikosituationen hingegen ist das Hornhaut-Bett vaskularisiert, z.B. infolge einer Hornhaut-Infektion oder einer Entzündung einer oder mehrerer Schichten der Hornhaut. Zusätzlich können pathologische Veränderungen der Vorderkammer, z.B. vordere Synechien, ein Glaukom oder eine chronische Instabilität des Tränenfilms, mit einer Beeinträchtigung des Transplantatüberlebens assoziiert sein. Definitionsgemäß ist jede Retransplantation nach immunvermitteltem Transplantatversagen eine Hochrisikokeratoplastik. Während risikoarme Keratoplastiken Erfolgsquoten von über 90\% nach 2 Jahren vorweisen können [3], kann bei der Hochrisikokeratoplastik die Abstoßungsrate bis zu 70\% betragen $[4,5]$. Hierbei ist bemerkenswert, dass diese hohen Ab- stoßungsraten auch bei starker topischer und systemischer Immunsuppression auftreten.

\section{Historischer Hintergrund}

Die erste perforierende Keratoplastik (pKP) wurde 1906 von Eduard Zirm durchgeführt [6]. Interessanterweise wurden die ersten Publikationen zu Transplantatabstoßung mehr als 40 Jahre später veröffentlicht, von Paufique [7] und Maumenee [8]. In der Zwischenzeit hatte man beobachtet, dass Allotransplantate solider Organe mit höherer Wahrscheinlichkeit versagten als HornhautAllotransplantate. Diese Beobachtung führte zu der Auffassung der Hornhaut als immunprivilegiertes Gewebe [8]. In den 1970er Jahren entdeckten Silverstein und Khodadoust Antigene, die von Hornhaut-Transplantaten nach der Transplantation freigesetzt wurden, und wiesen nach, dass der Empfänger auf diese Antigene mit einer Immunantwort reagieren kann. Dieser Prozess kann zur Abstoßung des Hornhaut-Transplantats führen [9, 10]. Die Neovaskularisation des Hornhaut-Betts ist mit der Herabsetzung des Immunprivilegs und dadurch mit vermehrtem Transplantatversagen aufgrund von Immunreaktionen verbunden.

\section{Histokompatibilitätsantigene}

Genau wie bei der Verpflanzung von soliden Organen exprimiert jedes Transplantat ein individuelles, charakteristisches Muster

\section{KARGER \\ Fax +497614520714 \\ information@karger.com}

www.karger.com (c) 2016 S. Karger GmbH, Freiburg

2297-0118/16/0021-0007\$39.50/0

Accessible online at:

www.karger.com/kop
Prof. Dr. med. Friedrich E. Kruse

Augenklinik

Universität Erlangen-Nürnberg

Schwabachanlage 6, 91054 Erlangen, Deutschland

friedrich.kruse@uk-erlangen.de 
von Molekülen auf der Oberfläche des Gewebes, die auch als «Transplantationsantigene» bezeichnet werden. Da einige dieser Transplantationsantigene eine stärkere Immunantwort auslösen als andere, wird zwischen «Haupt-» und «Nebenantigenen» unterschieden [11]. Ein zufällig ausgewähltes Transplantat bedeutet somit die Verpflanzung eines unterschiedlichen Musters von (allogenen) Transplantationsantigenen. Daher erkennt der Organismus des Empfängers das transplantierte Gewebe als körperfremd und leitet eine Immunantwort ein.

Die «Haupt»-Transplantationsantigene werden auch als humane Leukozytenantigene (HLA) bezeichnet; die für sie kodierenden Gene sind im Haupthistokompatibilitätskomplex (major histocompatibility complex; MHC) lokalisiert. Diese «Haupt»-Transplantationsantigene oder HLA werden in 2 Klassen eingeteilt, Klasse I und Klasse II. Klasse I umfasst die Transmembranglykoproteine HLA-A, HLA-B und HLA-C, die in zellspezifisch unterschiedlichen Mengen auf den meisten kernhaltigen Zellen (z.B. corneale Endothelzellen, Keratozyten und Epithelzellen) und Thrombozyten exprimiert werden. Die Antigene der Klasse II kommen auf der Oberfläche bestimmter Immunzellen vor, z.B. auf antigenpräsentierenden Zellen, Langerhans-Zellen des Epithels, Makrophagen und B-Lymphozyten. HLA-D-Gene regulieren typischerweise die Expression dieser Antigene (HLA-DP, HLA-DQ und HLA-DR) [12].

Die «Neben»-Transplantationsantigene werden an Genorten außerhalb des MHC kodiert, die im gesamten Genom verteilt sind. Nur wenn sie durch MHC-Klasse-I- oder -Klasse-II-Moleküle aufbereitet werden, erscheinen die Transplantationsantigene der Nebengruppe an der Oberfläche der allogenen Zellen. Ein Beispiel für diese «Neben»-Transplantationsantigene sind die AB0Blutgruppen-Antigene, die auch von Epithelzellen der Cornea exprimiert werden.

\section{Risikofaktoren für Hochrisikotransplantationen}

\section{Neovaskularisation der Hornhaut}

Die Neubildung von Blutgefäßen im Hornhaut-Bett des Transplantatempfängers ist der wichtigste Risikofaktor, der den Erfolg einer Hornhaut-Transplantation bedroht. Zahlreiche Autoren haben nachgewiesen, dass avaskuläre Hornhaut-Betten mit signifikant höheren Transplantat-Überlebensraten assoziiert sind als vaskularisierte $[13,14]$. Den «Collaborative Corneal Transplantation Studies» zufolge ist eine Hochrisikosituation durch eine Vaskularisierung von 2 oder mehr Quadranten definiert [15]. Wenn alle 4 Quadranten eine Gefäßbildung zeigten, war die Transplantat-Überlebensrate um rund $50 \%$ geringer. Interessanterweise wirkte sich die Vaskularisierung auch auf die Wahrscheinlichkeit einer endothelialen Abstoßung aus - etwa jeder dritte Patient mit einem vaskularisierten Hornhaut-Bett zeigte Anzeichen einer endothelialen Immunabstoßung. Von den Patienten ohne oder mit geringfügiger Neovaskularisation vor der Keratoplastik zeigte hingegen nur jeder siebte Patient eine solche endotheliale Abstoßungsreaktion [13]. Auch die Tiefe der neu gebildeten Hornhaut-Gefäße ist von Bedeutung: Tiefe, stromale
Neovaskularisationen führen zu einer höheren Transplantat-Abstoßungsrate als eine oberflächliche Neovaskularisation der Hornhaut $[15,16]$.

Das Ausmaß der Gefäßneubildung beeinflusst auch, wie lange es im Durchschnitt dauert, bis eine transplantierte Hornhaut Anzeichen von Abstoßung zeigt. In hochgradig neovaskularisierten Hornhaut-Betten betrug der Zeitraum ohne solche Komplikationen lediglich 2 Monate. Bei geringfügiger Neovaskularisation war dieser Zeitraum 4 Monate lang, und Empfänger mit gefäßfreier Hornhaut blieben nach dem Eingriff sogar durchschnittlich 10 Monate frei von Abstoßungssymptomen [17].

Auch die Erfolgsaussichten der Behandlung nach einer Hornhaut-Abstoßungsreaktion hängen mit der pathologischen Gefäßbildung im Hornhaut-Bett des Transplantatempfängers zusammen. Wenn Hornhaut-Transplantate in ein avaskuläres Hornhaut-Bett eingepflanzt werden, lassen sich rund zwei Drittel der Fälle erfolgreich behandeln, während bei Patienten mit präoperativ vaskularisierter Hornhaut nur jede zweite Hornhaut-Abstoßung behoben werden kann [18].

\section{Pathologien der Augenoberfläche}

Erkrankungen der Augenoberfläche gefährden die Integrität der transplantierten Spender-Hornhaut. Anomalien der Lider, der Tränendrüsen oder der Drüsen, die den Tränenfilm unterstützen, beeinträchtigen die Stabilität des Tränenfilms und dadurch die Nährstoffversorgung der Spender-Hornhaut [19]. Die Behebung pathologischer Zustände des Augenlids (z.B. Entropium) und die Verbesserung des Tränenfilms (z.B. durch Einsetzen eines Meatuspfropfs in das obere und/oder untere Punctum lacrimale bzw. eventuell deren Kauterisation) sind vor jeder Hornhaut-Operation von größter Bedeutung.

Schwerere Erkrankungen der Augenoberfläche einschließlich chemischer Verletzungen führen oft zu Limbus-Stammzell-Insuffizienzen und gefährden die Integrität und das Überleben des Hornhaut-Transplantats; Gleiches gilt für systemische Erkrankungen wie das Stevens-Johnson-Syndrom oder vernarbendes Pemphigoid. In diesen Fällen ist eine Hornhaut-Transplantation entweder kontraindiziert oder erfordert eine spezifische Vorgehensweise. Dieses Vorgehen kann in der Wiederherstellung der Augenoberfläche bestehen, z.B. mittels keratolimbaler Allotransplantation [20]. Allerdings werden hierzu hohe Hornhaut-Abstoßungsraten berichtet - unabhängig davon, ob die keratolimbale Allotransplantation und die pKP ein- oder zweizeitig durchgeführt werden. Eine andere Option ist die limbale Keratoplastik [21]. Bei diesem Eingriff, bei dem die Überlebensraten erheblich höher sind als bei der herkömmlichen pKP, wird vitales SpenderLimbusgewebe auf die Augenoberfläche transplantiert, nachdem eine oberflächliche lamelläre Keratektomie vorgenommen wurde (bis zu 65\% bei der Nachuntersuchung nach 5 Jahren, abhängig von der Anzahl Mismatches des Transplantats).

\section{Erneute Keratoplastik}

Nach dem Versagen einer früheren pKP infolge immunvermittelter Transplantatabstoßung ist die Prognose jeder nachfolgenden 
durchgreifenden Transplantation erheblich schlechter [14, 22]. Die Transplantat-Überlebensrate bei einem vaskularisierten Hornhaut-Bett beträgt nach einer ersten Transplantatabstoßung etwa $60 \%$, nach Abstoßung von 2 Hornhaut-Transplantaten noch $32 \%$ und nach Abstoßung eines dritten Transplantats weniger als $20 \%$ [17]. Interessant ist hierbei, dass eine Korrelation zwischen dem Zeitpunkt des Auftretens und der Schwere der Abstoßungsreaktion besteht [15].

\section{Größe des Hornhaut-Transplantats}

Der Durchmesser eines Hornhaut-Transplantats trägt zum Risiko des Transplantatversagens bei. Zahlreiche Autoren haben nachgewiesen, dass die Verpflanzung sehr großer Hornhaut-Lappen mit einem Anstieg des Transplantat-Abstoßungsrisikos einhergeht. Dies ist darauf zurückzuführen, dass mit der Größe des Transplantats auch die Zahl der in den Organismus des Empfängers eingebrachten Antigene zunimmt. Außerdem ist der Abstand zwischen dem vaskularisierten Limbus und der allogenen Hornhaut dann geringer als bei Transplantaten normaler Größe [22, 23].

Intraokulare Chirurgie, Entzündung des vorderen Segments Chirurgische Eingriffe gleichzeitig mit oder im Vorfeld einer pKP gehen mit einem erhöhten Risiko eines Transplantatversagens einher. Die Vitrektomie, Lentektomie oder chirurgische Eingriffe zur Senkung des Augeninnendrucks sind als Faktoren identifiziert worden, die zur Transplantatabstoßung beitragen, da physiologische Prozesse in der vorderen und/oder hinteren Kammer unterbrochen werden. Daher können vordere Synechien die Wahrscheinlichkeit eines Transplantatversagens um den Faktor 2 erhöhen, wenn 3 oder mehr Quadranten von Irisadhäsion betroffen sind [14, 24]. Die zugrunde liegende Ursache hierfür scheint der Verlust des Immunprivilegs des allogenen Transplantats zu sein [25]. Es könnten aber auch Scherkräfte an der Rückfläche der Hornhaut zum Verlust cornealer Endothelzellen führen [26]. Ein erhöhter Augeninnendruck wirkt sich negativ auf die Überlebensfähigkeit des Endothels aus [26]; dies verstärkt den Zellverlust. In ähnlicher Weise ist bei einer pKP, die im akuten Stadium einer Entzündung des vorderen Segments vorgenommen wird, die Wahrscheinlichkeit des Transplantatüberlebens verringert. Sofern sie nicht zwingend notwendig ist (z.B. als Notfallkeratoplastik bei Hornhaut-Perforation), ist eine solche Transplantation um 6-12 Monate aufzuschieben, bis sich der Zustand in der Vorderkammer beruhigt hat [27].

Verschiedene systemische Erkrankungen, wie z.B. das StevensJohnson-Syndrom, okuläres vernarbendes Pemphigoid, Uveitis oder eine atopische Erkrankung, gehen mit einer schlechten Prognose für die pKP einher. Betroffene Patienten müssen interdisziplinär behandelt werden, gegebenenfalls mit systemischer Immunsuppression. Offene Gespräche mit dem Patienten sind entscheidend, um die Kooperation nach dem Eingriff sicherzustellen.

\section{Herpes simplex}

Weithin ist anerkannt, dass durch Herpes simplex hervorgerufene Keratitis einen negativen prognostischen Faktor für die pKP darstellt [28, 29]. Die Inzidenz von Transplantatversagen ist hier signifikant höher als bei konventionellen risikoarmen Keratoplastiken (z.B. bei Keratokonus); bis zu 70\% der Transplantatabstoßungen sind nicht reversibel [30]. Ein Grund für diese hohe Rate irreversiblen Transplantatversagens ist möglicherweise die klinische Ähnlichkeit regulären Transplantatversagens mit Herpes-simplex-Infektionen [30]. Die prophylaktische Anwendung systemischer antiviraler Mittel vermag zwar, die Herpes-Rezidivrate zu senken [26], doch eine pKP nach Herpes-simplex-Infektion ist dennoch weiterhin in hohem Maße von Immunabstoßung bedroht. Zusätzlich zum erhöhten immunologischen Risiko sind infizierte Patienten auch von neurotrophen Störungen der Hornhaut-Oberfläche betroffen, die häufig zu einer gestörten Regeneration der Augenoberfläche führen.

\section{Alter des Patienten}

Das Abstoßungsrisiko nach einer Hornhaut-Transplantation ist umso höher, je niedriger das Alter des Patienten ist. Besonders ausgeprägt ist dies bei Kindern - bei Patienten unter 15 Jahren erreicht die Inzidenz des Transplantatversagens innerhalb der ersten 2 Jahre 30-40\%. Im Vergleich zu einem älteren Patientenkollektiv ist bei Patienten unter 40 Jahren das Abstoßungsrisiko doppelt so hoch wie das Risiko des Transplantatversagens [31]. Ein Grund für diese Problematik könnte darin liegen, dass jüngere Spender mehr HLA exprimieren, wodurch das Transplantat mit höherer Wahrscheinlichkeit vom Immunsystem des Empfängers erkannt wird [32].

\section{Postoperatives Management der Hochrisikokeratoplastik}

Bei der Behandlung von Hochrisiko-Keratoplastikpatienten ist hinsichtlich der topischen und systemischen Anwendung von Therapeutika besonders aufmerksam vorzugehen.

Größtenteils besteht Einigkeit dahingehend, dass Konservierungsmittel-freie topische Antibiotika und Befeuchtungsmittel anzuwenden sind, bis das Hornhaut-Epithel geschlossen ist. Die Anwendung von Steroiden vor dem epithelialen Wundverschluss ist zunächst optional, wird aber notwendig, sobald die Hornhaut-Epithelzellen eine intakte und stabile Epithelschicht gebildet haben.

\section{Behandlung von Patienten mit vorhergehender Herpes-simplex-Infektion}

Vor der pKP wird eine prophylaktische orale Dosis von $5 \times 200 \mathrm{mg}$ Aciclovir täglich empfohlen. Postoperativ sollte diese Dosis auf $5 \times 400 \mathrm{mg}$ oral für die Dauer der Behandlung mit systemischen Steroiden erhöht werden [33,34], unterstützt durch topische Aciclovir-Salbe.

Bei Patienten mit erhöhtem Risiko für ein erneutes Auftreten einer Herpes-simplex-Keratitis ist eine Dosierung von 800-1000 mg täglich für 6-12 Monate erforderlich [35, 36]. In Fällen mit hohem Risiko für eine immunvermittelte Abstoßung und wiederkehrende Herpes-Keratitis ist zusätzlich Mycophenolat-Mofetil (MMF) $2 \times 1$ g täglich für 6-12 Monate zu verabreichen [37]. 
Systemische Steroide sind anfänglich in einer Dosierung von 1-2 mg/kg Körpergewicht täglich anzuwenden und dann allmählich auszuschleichen, sodass eine Gesamtbehandlungsdauer von rund 3 Wochen erreicht wird [38].

\section{Systemische Behandlung von Hochrisikopatienten und Human-Leukozyten-Antigen-Matching}

Um die Überlebensraten von Hornhaut-Transplantaten in Hochrisikosituationen $\mathrm{zu}$ verbessern, muss die vorstehend beschriebene Basistherapie mit Steroiden um stärkere Immunsuppressiva erweitert werden (z.B. Cyclosporin A, MMF). Diese ersetzen mittelfristig die Kortikosteroide und sorgen für langfristige Immunsuppression. In allen Fällen, in denen systemische Immunsuppressiva angewandt werden, muss ein Facharzt für Innere Medizin hinzugezogen werden, um das Gesamtrisiko dieser Form der Immunsuppression zu beurteilen. Leber, Nieren, Blut und Blutdruck des Patienten müssen gründlich untersucht werden, da Cyclosporin A zu Hypertonie und Nierentoxizität und MMF zu Lebertoxizität und gastrointestinalen Beschwerden führen können.

Cyclosporin A wird in einer Dosierung von 3-5 mg/kg Körpergewicht pro Tag verschrieben, wobei die Cyclosporin-Konzentration im Blut überwacht wird (therapeutische Konzentration 100150 ng/ml; ermittelte Konzentration variiert stark je nach Labor und Zeitpunkt der Arzneimittel-Gabe). Die Dosierung muss nach Maßgabe der Cyclosporin-Serumkonzentration angepasst werden. Die Dauer der Behandlung richtet sich nach dem individuellen Transplantat-Abstoßungsrisiko des Patienten [39].

MMF kann bei Kontraindikationen gegen Cyclosporin A eingesetzt werden, außerdem in Kombination mit Aciclovir nach Keratoplastik bei Patienten mit Herpes-simplex-Keratitis. Die empfohlene Dosis beträgt $2 \times 1 \mathrm{~g}$ täglich. Die Wahl von MMF als Immunsuppressivum hat den Vorteil, dass keine regelmäßige Kontrolle der Blutserum-Konzentration erforderlich ist.

Eine wichtige Maßnahme, die sich seit einigen Jahren etabliert, ist das HLA-Matching von Spender und Empfänger. Dabei wird das Blut des Patienten auf seinen HLA-Phänotyp untersucht; dieser wird dann an große Augenbank-Netzwerke gemeldet, um so schnell wie möglich passendes Spendergewebe zu finden. Da in manchen Augenbanken auch der HLA-Phänotyp der Spendergewebe bestimmt wird, können passende Spender-Empfänger-Paare gebildet werden. Das verbessert die Chancen für weniger Transplantatabstoßung bei Hochrisikokeratoplastiken [39]. Aufgrund der Verschiebung der Indikation der pKP hin zur lamellierenden Keratoplastik (insbesondere zur Descemet-Membran-Endothel-Keratoplastik (DMEK) mit ihren sehr geringen Abstoßungsraten) nimmt die frühere Relevanz der HLA-Typisierung derzeit in gewissem Maße ab und ist heute auf pKP-Retransplantationen mit sehr hohem Risiko beschränkt [40].

\section{Risiken der lamellierenden Keratoplastik}

\section{Die Rolle der posterioren lamellierenden Keratoplastik}

Durch die Entwicklung neuer Techniken für die vordere und hintere lamelläre Chirurgie ist die schichtspezifische Behandlung von Hornhaut-Erkrankungen möglich geworden. Nachdem die durchgreifende Keratoplastik annähernd 100 Jahre lang der Goldstandard war, geht die Zahl dieser Eingriffe heute zurück, während die Zahl der lamellierenden Hornhaut-Operationen kontinuierlich zunimmt.

Endotheliale Pathologien wie die Fuchs-Endotheldystrophie machen den Großteil der klinischen Fälle in Augenkliniken in Europa und Amerika aus. Der Ersatz der Descemet-Membran mittels «Descemet stripping automated endothelial keratoplasty» (DSAEK) oder DMEK etabliert sich derzeit in der augenchirurgischen Routinepraxis [41].

Da das Hornhaut-Stroma und -Epithel als Hauptträger der Antigenmenge gelten, die die Immunantwort nach der Transplantation auslöst, ist zu erwarten, dass die Einpflanzung eines lamellären Allotransplantats mit weniger (bei der DSAEK) bzw. gar keinem Stroma (bei der DMEK) eine geringere Immunreaktion hervorruft. Tatsächlich verzeichneten Anshu und Mitarbeiter für die DSAEK im Vergleich zur pKP einen Rückgang der Transplantat-Abstoßungsrate bei der 1- und 2-Jahres-Nachbeobachtung um den Faktor 15; im Fall der DMEK erzielten sie, verglichen mit der pKP, bei Niedrigrisikopatienten sogar einen Rückgang um den Faktor 20 [42]. Nach der DMEK traten in 1\% der Fälle AbstoBungsreaktionen auf (sowohl nach 1 als auch nach 2 Jahren), nach der DSAEK in $8 \%$ der Fälle nach 1 Jahr und in 12\% nach 2 Jahren. Nach der pKP waren im Vergleich dazu mit 14\% bzw. 18\% erheblich höhere Abstoßungsraten zu verzeichnen [42].

Interessanterweise scheint das antigene Potenzial von DMEKTransplantaten zur Induktion einer klinisch relevanten Immunantwort des Empfängers recht begrenzt zu sein. Es wurde berichtet, dass eine um den Faktor 10 niedrigere Kortikoidkonzentration (Prednisolonacetat $1 \%$ vs. Fluormetholon 0,1\%) immer noch ausreichend ist, um $1 \mathrm{Jahr}$ nach der DMEK-Transplantation eine Abstoßungsrate von $1 \%$ zu erreichen [43].

\section{Die Rolle der anterioren lamellierenden Keratoplastik}

Die anteriore Keratoplastik ist als teilweiser Ersatz für die pKP in Indikationen mit verbleibendem physiologischem Hornhaut-Endothel gebräuchlich (z.B. bei Keratokonus oder Hornhaut-Narben) [44]. Da die Operation selbst technisch anspruchsvoll ist und die erfolgreiche Trennung der Descemet-Membran vom Stroma eine Herausforderung darstellt, ist die Zahl der Chirurgen, die in diesen Fällen eine konventionelle pKP durchführen, weiterhin groß [45]. In einer Meta-Analyse wurde die tiefe lamellierende Keratoplastik (deep anterior lamellar keratoplasty; DALK) mit der pKP verglichen. Wie erwartet war die Zahl der Endothelzellen bei den DALK-Patienten signifikant höher, und auch das resultierende Sehvermögen war besser. Die Rate von Transplantatversagen und der postoperative Astigmatismus waren jedoch in beiden Gruppen vergleichbar. Bei den DALK-Patienten traten weniger Komplikationen auf, z.B. keine expulsiven Blutungen und keine Endophthalmitis [46]. Diese Ergebnisse sind jedoch kontrovers, da andere Autoren in einer Meta-Analyse zu DALK versus pKP in Bezug auf Keratokonus keine signifikanten Unterschiede feststellen konnten [47]. Langzeitstudien werden klären, von welcher chi- 
rurgischen Strategie der Patient stärker profitiert, unter Berücksichtigung sowohl des Retransplantationsrisikos als auch des resultierenden Sehvermögens.

\section{Ausblick}

Die Hochrisikokeratoplastik stellt sowohl für chirurgische als auch für konservative Ophthalmologen nach wie vor eine große
Herausforderung dar. Doch Fortschritte in der Immunsuppressionstherapie könnten in der Zukunft neue Wege zur Vorbeugung und Behandlung von Immunreaktionen auf Allotransplantate eröffnen. Der Wandel auf dem Gebiet der Ophthalmochirurgie insbesondere in Form der Abwendung von der pKP hin zur hinteren lamellierenden Keratoplastik - bedeutet einen echten Durchbruch für die Patienten. In einigen Indikationen lässt sich das Risiko der Immunabstoßung nach DMEK nahezu ausmerzen.

\section{Referenzen}

1 European Eye Bank Association, Venice, Italy, 2010.

2 Eye Bank Association of America, Washington, DC, 2015.

$\checkmark 3$ Council on Scientific Affairs: Report on the organ transplant panel: corneal transplantation. JAMA 1988;259:719-722.

4 Mader TH, Stulting RD: The high-risk penetrating keratoplasty. Ophthalmol Clin North Am 1991;4:411-426.

5 Foulks GN, Sanfilippo F: Beneficial effects of histocompatibility on high-risk corneal transplantation. Am J Ophthalmol 1982;94:622-629.

6 Zirm EK: Eine erfolgreiche totale Keratoplastik (A successful total keratoplasty). 1906. Refract Corneal Surg 1989;5:258-261.

7 Paufique L, Sourdille GP, Offret G (eds): Les Graffes de la Cornée. Paris, Masson, 1948.

$>8$ Maumenee AE: The influence of donor-recipient sensitization on corneal grafts. Am J Ophthalmol 1951;34:134-152.

$\checkmark 9$ Khodadoust AA, Silverstein AM: Transplantation and rejection of individual cell layers of the cornea. Invest Ophthalmol Vis Sci 1969;8: 180-195.

10 Khodadoust AA, Silverstein AM: Studies on the nature of the privilege enjoyed by corneal allografts. Invest Ophthalmol Vis Sci 1972;11: 137-148.

11 Klein J: Natural History of the Major Histocompatibility Complex. New York, NY, Wiley, 1986.

12 Streilein JW: Immunobiology and immunopathology of corneal transplantation. Chem Immunol 1999;73:186-206.

$\checkmark 13$ Alldredge OC, Krachmer JH: Clinical types of corneal transplant rejection. Arch Ophthalmol 1981;99:599-604.

14 Maguire MG, Stark WJ, Gottsch JD, et al: Risk factors of corneal graft failure and rejection in the collaborative corneal transplantation studies. Collaborative Corneal Transplantation Studies Research Group. Ophthalmology 1994; 101:1536-1547.

15 The Collaborative Corneal Transplantation Studies Research Group: The collaborative corneal transplantation studies (CCTS). Effectiveness of histocompatibility matching in high-risk corneal transplantation. Arch Ophthalmol 1992;110:1392-1403.
6 Polack FM: Scanning electron microscopy of corneal graft rejection: epithelial rejection, endothelial rejection, and formation of posterior graft membranes. Invest Ophthalmol 1972;11: $1-14$.

17 Khodadoust AA: The allograft rejection reaction: the leading cause of late failure of clinical corneal grafts; in Jones BR (ed): Corneal Graft Failure. New York, NY, Elsevier, 1972.

18 Fine M, Stein M: The role of corneal vascularization in human graft rejection; in Porter R, Knight J, Ciba Foundation (eds): Corneal Graft Failure. Amsterdam/New York, Associated Scientific Publishers, 1973, pp 193-204.

19 Nakamura S, Kinoshita S, Yokoi N, et al: Lacrimal hypofunction as a new mechanism of dry eye in visual display terminal users. PLoS One 2010;5:e11119.

20 Shimazaki J, Maruyama F, Shimmura S, et al: Immunologic rejection of the central graft after limbal allograft transplantation combined with penetrating keratoplasty. Cornea 2001;20: 149-152.

21 Reinhard T, Spelsberg H, Henke L, et al: Longterm results of allogeneic penetrating limbokeratoplasty in total limbal stem cell deficiency. Ophthalmology 2004;111:775-782.

22 Boisjoly HM, Bernard PM, Dube I, et al: Effects of factors unrelated to tissue matching on corneal transplant endothelial rejection. Am J Ophthalmol 1989;107:647-654.

23 Völker-Dieben HJ, D’Amaro J, Kok-van Alphen CC: Hierarchy of prognostic factors for corneal allograft survival. Aust N Z J Ophthalmol 1987;15:11-18.

24 Sit M, Weisbrod DJ, Noar J, et al: Corneal graft outcome study. Cornea 2001;20:129-133.

25 Yamagami S, Tsuru T: Increase in orthotopic murine corneal transplantation rejection rate with anterior synechiae. Invest Ophthalmol Vis Sci 1999;40:2422-2426.

26 Wilson SE, Kaufman HE: Graft failure after penetrating keratoplasty. Surv Ophthalmol 1990;34:325-356.

27 Nobe JR, Moura BT, Robin JB, et al: Results of penetrating keratoplasty for the treatment of corneal perforations. Arch Ophthalmol 1990; 108:939-941.
28 Coster DJ: Factors affecting the outcome of corneal transplantation. Ann R Coll Surg Engl 1981;63:91-97.

29 Moyes AL, Sugar A, Musch DC, et al: Antiviral therapy after penetrating keratoplasty for herpes simplex keratitis. Arch Ophthalmol 1994; 112:601-607.

30 Epstein RJ, Seedor JA, Dreizen NG, et al: Penetrating keratoplasty for herpes simplex keratitis and keratokonus. Allograft rejection and survival. Ophthalmology 1987;94:935-944.

31 Stulting RD, Sumers KD, Cavanagh HD, et al: Penetrating keratoplasty in children. Ophthalmology 1984;91:1222-1230.

32 Palay DA, Kangas TA, Stulting RD, et al: The effects of donor age on the outcome of penetrating keratoplasty in adults. Ophthalmology 1997;104:1576-1579.

33 Barney NP, Foster CS: A prospective randomized trial of oral acyclovir after penetrating keratoplasty for herpes simplex keratitis. Cornea 1994;13:232-236.

34 Kersten A, Sundmacher R, Reinhard T: Postoperative Komplikationen nach perforierender Keratoplastik in Herpesaugen. Differentialdiagnose, Therapie und prognostische Bedeutung. Ophthalmologe 1997;94:889-896.

35 Tambasco FP, Cohen EJ, Nguyen LH, et al: Oral Acyclovir after penetrating keratoplasty for herpes simplex keratitis. Arch Ophthalmol 1999;117:445-449.

>36 van Rooj J, Rijneveld WJ, Remeijer L, et al: Effect of oral acyclovir after penetrating keratoplasty for herpetic keratitis: a placebo-controlled multicenter trial. Ophthalmology 2003; 110:1916-1919.

37 Mayer K, Reinhard T, Reis A, et al: Synergistic antiherpetic effect of acyclovir and mycophenolate mofetil following keratoplasty in patients with herpetic eye disease: first results of a randomised pilot study. Graefe's Arch Clin Exp Ophthalmol 2003;214:1051-1054.

-38 Hill JC: Immunosuppression in corneal transplantation. Eye 1995;9:247-253.

$>39$ Reinhard T, Sundmacher R, Godehardt E, et al: Systemische Cyclosporin-A-Prophylaxe nach Keratoplastiken mit erhöhtem Risiko für Immunreaktionen als einzigem erhöhten Risikofaktor. Ophthalmologe 1997;94:496-500. 
40 Böhringer D, Ihorst G, Grotejohann B, et al: Functional antigen matching in corneal transplantation: matching for the HLA-A, -B and -DRB1 antigens (FANCY) - study protocol. BMC Ophthalmol 2014;14:156.

41 Rodríguez-Calvo-de-Mora M, Quilendrino R, Ham L, et al: Clinical outcome of 500 consecutive cases undergoing Descemet's membrane endothelial keratoplasty. Ophthalmology 2015; 122:464-470.

42 Anshu A, Price MO, Price FW Jr: Risk of corneal transplant rejection significantly reduced with Descemet's membrane endothelial keratoplasty. Ophthalmology 2012;119:536-540.
43 Price MO, Price FW Jr, Kruse FE, et al: Randomized comparison of topical prednisolone acetate $1 \%$ versus fluorometholone $0.1 \%$ in the first year after descemet membrane endothelial keratoplasty. Cornea 2014;33:880-886.

44 Sarnicola V, Toro P, Sarnicola C, et al: Longterm graft survival in deep anterior lamellar keratoplasty. Cornea 2012;31:621-626.

45 Kasbekar SA, Jones MN, Ahmad S, et al: Corneal transplant surgery for keratoconus and the effect of surgeon experience on deep anterior lamellar keratoplasty outcomes. Am J Ophthalmol 2014;158:1239-1246.
46 Liu H, Chen Y, Wang P, et al: Efficacy and safety of deep anterior lamellar keratoplasty vs. penetrating keratoplasty for keratoconus: a meta-analysis. PLoS One 2015;10:e0113332.

47 Keane M, Coster D, Ziaei M, et al: Deep anterior lamellar keratoplasty versus penetrating keratoplasty for treating keratoconus. Cochrane Database Syst Rev 2014;7:CD009700. 